\title{
Representaciones sociales sobre maternidad, paternidad, matrimonio y familia en adolescentes escolarizados de Bogotá (Colombia) ${ }^{1}$
}

Social representations on maternity, paternity, marriage and family in schooling teenagers of Bogotá (Colombia) ${ }^{2}$

Representações sociais sobre maternidade, paternidade, casamento e família em adolescentes escolarizados de Bogotá (Colômbia) ${ }^{3}$

\author{
María del Carmen Docal Millan 4 \\ Lina Adriana Gómez Cruz ${ }^{5}$ \\ Diana Milena Gutiérrez Casas ${ }^{6}$ \\ Diana Beatriz Jerez Guerrero ${ }^{7}$ \\ Recibido: 22.06.2016 • Arbitrado: 24.10.2016 • Aprobado:31.10.2016
}

\begin{abstract}
${ }^{1}$ Este artículo se deriva de un estudio de mayor escala, "Sentidos y percepciones sobre familia de jóvenes estudiantes de colegios distritales", código FAM-172015 financiado por el Instituto de La Familia de la Universidad de La Sabana realizado entre febrero del 2014 y abril de 2016.

${ }^{2}$ This article is deduced from a mayor scale study, "Senses and perceptions on young students family", code FAM-17-2015 financed by the Instituto de La Familia of Universidad de La Sabana, elaborated between february of 2014 and april of 2016.

${ }^{3}$ Este artigo deriva de um estudo de maior escala, "Sentidos e percepções sobre a família de jovens estudantes de colégios distritais", código FAM-172015, financiado pelo Instituto de laFamilia da Universidad de La Sabana, realizado entre fevereiro de 2014 e abril de 2016.

${ }^{4}$ Magister en Estudios Políticos de la Pontificia Universidad Javeriana, Bogotá - Colombia, Trabajadora Social de la Universidad Colegio Mayor de Cundinamarca, Bogotá - Colombia, Directora y profesora de la Maestría en Asesoría Familiar y Gestión de Programas para la Familia. Miembro del Grupo de investigación Familia y Sociedad de la Universidad de La Sabana, Chía, Colombia, maria.docal@unisabana.edu.co

${ }^{5}$ Magister en Asesoría Familiar y Gestión de Programas para la Familia de la Universidad de La Sabana. Especialista en Gerencia de Proyectos Educativos de la Universidad Cooperativa de Colombia, Bogotá, Colombia, Licenciada
\end{abstract}




\section{Resumen}

La presente investigación pretende conocer sobre las representaciones sociales que en materia de familia, maternidad, paternidad y matrimonio construyen los adolescentes escolarizados en colegios oficiales de Bogotá. Se presenta un estudio descriptivo transversal de metodología mixta mediante la participación de 2.535 hombres y mujeres adolescentes. Los resultados indican que para los participantes es importante terminar estudios de bachillerato y universitarios antes de fundar una familia y tener hijos. El 66\% considera la familia como unidad de amor, seguida por el lugar de la ayuda mutua e incondicional con el $\mathbf{1 4 . 6 \%}$. Respecto de si la maternidad y la paternidad son parte del desarrollo de la persona, el 63,98\% de los participantes en adolescencia temprana consideran que sí, frente al 36,4\% de adolescencia intermedia y al 15,2\% de los que están en adolescencia avanzada. El estudio concluye que existen diferencias por sexo frente a la maternidad y paternidad en el proyecto de vida.

Palabras clave: Adolescencia, familia, maternidad, paternidad, matrimonio, representaciones sociales.

\section{Abstract}

This research pretends to know about the social representations that in terms of family, maternity, paternity and marriage create the teenagers that attend official schools in Bogotá. We present a study with cross curricular descriptive methodology with the participation of 2.535 adolescents between males and females. The results show that, for the participants, it's important to finish school and university studies before settling down. $66 \%$ consider the family a love unit, followed

en Psicología y Pedagogía de la Universidad Pedagógica Nacional, Bogotá, Colombia, linagocr@unisabana.edu.co

${ }^{6}$ Magíster en Asesoría Familiar y Gestión de Programas para la Familia de la Universidad de La Sabana. Licenciada en Educación con Énfasis en Educación Especial, Universidad Pedagógica Nacional, Bogotá, Colombia, Especialista en Aprendizaje Escolar y sus Dificultades de la Universidad Cooperativa de Colombia, Bogotá, Colombia,diangutca@unisabana.edu.co 
by a place of mutual and unconditional help with a 14.6\%. Concerning if maternity and paternity are an important part of personal growth, $63.98 \%$ of young adolescent participants consider it is, compared to $36,4 \%$ of the older adolescents and the $15.2 \%$ of the oldest adolescents. The study concludes that there are differences in gender towards maternity and paternity in their life project.

Keyword: Adolescence, family, maternity, paternity, marriage, social representations

\section{Resumo}

A presente pesquisa pretende saber sobre as representaçõessociais que, comrelação a família, maternidade, paternidade e casamento, constroem os adolescentes escolarizados emcolégiosoficiais de Bogotá. Apresenta-se umestudodescritivo transversal de metodologia mista com a participação de 2.535 homens e mulheres adolescentes. Os resultados indicam que, para os participantes, é importante terminar os estudos do ensinomédio e universitários antes de formar umafamília e ter filhos. $66 \%$ deles considera a família como unidade de amor, conceito seguido por lugar de ajudamútua e incondicional, com 14,6\%. Comrespeito à suposição de se a maternidade e a paternidadesão parte do desenvolvimentopessoal, 63,98\% dos participantes adolescentes maisjovensconsideram que sim, ante $36,4 \%$ dos adolescentes emidadeintermediária e 15,2\% dos adolescentes emidademaisavançada. O estudoconclui que existemdiferenças por sexo perante a maternidade e a paternidade no projeto da vida.

Palavras-chave: Adolescência, família, maternidade, paternidade, casamento, representaçõessociais. 


\section{Introducción}

La familia como tema axial de la humanidad a través de la historia ha sido de interés de las ciencias humanas y sociales. Es considerada como el primer grupo en el que se brindan las herramientas para el desarrollo personal, social y cultural del individuo persona. Igualmente, se reconoce que los cambios culturales, sociales y económicos de la sociedad la han afectado, sin embargo sigue siendo concebida como el apoyo fundamental de la persona y el escenario principal de formación del sujeto social (Donati, 2003, 2014; Pérez-Adán, 2005; Rodríguez, 2012; Hadjadj, 2015).

En esta línea de pensamiento, el proceso de socialización incluye el legado cultural que en materia de familia está representado entre otros aspectos por las creencias, valores y comportamiento sobre ser hombre, mujer, padre, madre, pareja, ser y hacer familia. No obstante, los sistemas sociales cambian en el tiempo y según los contextos sociales e históricos (Castro, Rojas, Pinilla y Docal, 2015).

En este sentido, Bronfenbrenner (1987), desde la teoría ecológica, comprende la continua acomodación de la persona y las propiedades cambiantes de los distintos contextos en los que se desarrolla la vida cotidiana y en este proceso reconoce las mutuas influencias entre los contextos y la persona. La presente investigación se centra en el contexto familiar, en tanto allí se inicia el proceso de socialización.

De otra parte, las representaciones sociales de la maternidad y la paternidad, se entienden desde un significado reflexivo, habitual y automatizado. Para Moscovici (1979), la importancia de las representaciones sociales se ubica en su naturaleza implícita y en la poca conciencia que tiene la persona de su existencia; al esta ser menor, más fuertes son las vivencias individuales y el lugar que ocupa en su medio, y las situaciones diarias confluyen en su formación individual, en su forma de percibir y vivir el mundo.

El mismo autor determina tres dimensiones que hacen parte de las diferentes culturas, constituyendo un universo de creencias y prác- 
ticas porque estas dimensiones y conceptualizaciones se relacionan con la disposición de los conocimientos que los grupos sociales construyen respecto a un objeto social determinado. A la primera corresponde la información adquirida y acumulada a través del tiempo. La segunda, a la actitud como la intención positiva o negativa que se atribuye a la representación y la tercera, al campo de imagen que da valor y precisión del objeto que varía entre grupos. Así, las representaciones sociales facilitan la adaptación de las personas al entorno y a la internalización de sus experiencias.

En este mismo sentido, Van Dijk (1999) define las representaciones sociales como el conjunto de creencias y sistemas básicos de la cognición social compartidos y específicos a un grupo particular, los cuales se ubican dentro de las creencias generales como valores, opiniones, conocimiento y criterios de verdad. Moscovici (1984), en este mismo sentido, planteó el carácter plástico de las representaciones sociales en tanto adquieren formas dinámicas.

Ahora bien, respecto a la adolescencia, varios autores la entienden como una etapa de la vida de cambios a nivel biológico, cognitivo y afectivo de forma continua, profunda y compleja en la biografía de las personas (Erickson, 1968; Mardomingo, 1994). Lalueza y Crespo (2003) afirman que "los cambios cognitivos permiten al adolescente evaluar la realidad a partir de diferentes puntos de vista, de imaginar otros modos de funcionamiento y de elaborar respuestas propias" (p. 138), lo que permite la construcción de su propia identidad personal y cultural.

Papalia (2005) afirma que se da inicio a la adolescencia desde la pubertad con cambios físicos, psicológicos y sociales, además de ser "el proceso por el cual una persona alcanza la madurez y la capacidad para reproducirse" (p. 359). Es decir, inicia entre los 11 y 12 años de edad y culminaa los 18 o 19 años. Para efectos del estudio actual se acoge la clasificación que plantea Papalia (2005) en las tres etapas, así: la adolescencia temprana en el rango de edades entre los 12 a 14 años de edad, en la que el pensamiento del adolescente es más abstracto, analítico y autocrítico; es una persona que se expresa más libre y reflexiva 
asumiendo una postura egocéntrica. La adolescencia intermedia corresponde a las edades comprendidas entre los 15 y 16 años, tiempo en la que se da un periodo de adaptación y aceptación de sí mismo al reconocer características propias, y la adolescencia tardía, entre los 17 a 18 años de edad; "se caracteriza por la mayor autonomía e independencia de la adolescente, por los cambios emocionales menos marcados, menor ambivalencia y egocentrismo" (Papalia. 2005. P.391).

De otra parte, para Alpízar y Bernal (2003) existen elementos predominantes desde las diferentes perspectivas teóricas sobre adolescencia y juventud, entre las cuales se destaca la característica homogenizante que valora a los adolescentes con las mismas necesidades o visiones del mundo y la característica estigmatizante, que considera la adolescencia como un ciclo vital de crisis y de problemas, y por tanto, es percibida como riesgosa.

En esta línea de pensamiento Gómez, Molina y Zamberlin (2011) plantean que los adolescentes están permanentemente expuestos en el contexto social y en los medios de comunicación a mensajes erotizantes y a discursos que promueven la liberación sexual que se asocian al inicio temprano de la actividad sexual. Igualmente plantean que en Latinoamérica con patrones culturales tan tradicionales la sexualidad en los adolescentes no es tan aceptada, generando dificultades en la educación desde la familia sobre este tema y en sí sobre la vida familiar.

Ahora bien, la sexualidad en la adolescencia como objeto de interés de las ciencias sociales y de los gobiernos se ha centrado en el embarazo adolescente por el impacto social, cultural y en el proyecto de vida de los jóvenes (Raneri y Wiemann, 2007; Nóblega, 2009; Galindo, 2012; Unicef, 2014; Cabrera et al., 2014).

Sin embargo, se encuentran estudios que han centrado su atención en la construcción de sentido y de las representaciones sociales de los adolescentes en relación con la maternidad y paternidad, es el caso de la investigación de Lerner, Noh yWilson (1998) en Estados Unidos, quienes estudiaron la importancia de la crianza de los adolescentes y 
su influencia en su futuro como padres y concluyeron que asumir este papel implica una resignificación del rol.

Los estudios en Brasil de Bonfá, Menandro y Cristina (2014) y de Barreto (2013), sobre las representaciones sociales en hombres adolescentes sobre la paternidad, abren un campo de estudio para avanzar en la comprensión de las ideas que sobre la maternidad y la paternidad construyen los adolescentes. Bonfá, Menandro y Cristina (2014) plantean que "un aspecto que merece ser añadido en las investigaciones futuras es incluir la percepción de los jóvenes sobre su propia crianza, lo que le permitiría originar datos más enriquecidos, así como matices más explícitos de estas representaciones de los elementos que los jóvenes han anunciado"(p. 322).

En esta línea de pensamiento, Barreto (2013) realizó un estudio con adolescentes varones sobre paternidad, y recomendó involucrar a la madre y al padre adolescente por las implicaciones que tiene el fenómeno en sus vidas y en su cultura.

En Perú, Nóblega (2009), a partir de su investigación, planteó que las adolescentes encuentran en la maternidad temprana una forma de escapar de situacionesfamiliares adversas como la falta de afecto, adicciones o conductas inapropiadas de los padres. Igualmente, algunas adolescentes ven de forma positiva la maternidad porque pueden transformar estas situaciones cuando asumen la responsabilidad del cuidado y crianza de su hijo, no obstante las dificultades que se representan por su edad.

Por su parte, Galindo (2012) estudió el embarazo y la maternidad adolescente, las causas y consecuencias de la maternidad temprana en seis regiones de Colombia y concluyó que "el incremento de los embarazos en la adolescencia se debe a circunstancias como el nivel educativo, el estado civil, el contexto familiar y social que rodea a las adolescentes afectando directamente las perspectivas sobre su futuro" (p.135).

El estudio de Cabrera, Salazar, Docal, Aya, Ardila y Rivera (2014) avanza al estudiar algunos contextos individuales, familiares y esco- 
lares que permiten predecir el inicio de la actividad sexual en adolescentes colombianos y plantean que en la medida en que avanzan en edad, van consolidando sus ideas sobre amor, afectividad y sexualidad y sugieren que se posponga el inicio de la actividad sexual a fin de fundamentar ideas sobre el tema, de tal manera que tomen decisiones reflexivas y autónomas.

Ahora bien, es importante entender que la maternidad y la paternidad ha cambiado desde la mitad del Siglo XX producto del ingreso de la mujer a la fuerza laboral, el uso de métodos anticonceptivos, la emergencia de formas de composición familiar, cambios en los roles del hombre y la mujer dentro del hogar, el acceso masivo a medios de comunicación, entre otros factores. Maldonado y Micolta (2003) afirman que "han roto con algunas maneras de "ser padre" o "ser madre" de la década de los 50 o 60, dando así lugar a diversas tendencias de la parentalidad” (p. 101). Sin embargo, como lo plantea Llanio (2009) la maternidad sigue siendo un referente de afirmación de la feminidad, no obstante, no es la primera ni la única expectativa para las mujeres.

Puyana y Mosquera (2005) afirman que al estudio de los fenómenos de la maternidad y paternidad, además de agregarse un factor como las relaciones de género, es necesario revisar otros igualmente transversales como las diferenciaciones sexuales y las representaciones sociales que configuran sentidos y significados al ejercicio de ser madre o padre dentro de un contexto cambiante y dinámico.

En la vida cotidiana social y familiar se expresa la complejidad de los cambios que se han produciendo en torno a los asuntos relacionados con la paternidad y de la maternidad. Para las ciencias sociales, en el mundo moderno es importante estudiar, desde otras perspectivas, el fenómeno del embarazo adolescente con la integración de métodos cuantitativos y cualitativos que permitan avanzar en la comprensión de las formas de entender y asumir la maternidad y la paternidad dentro de la complejidad que se impone en cuanto a la formación de las generaciones presentes y futuras. 
Aunque, como ya se anotó, la literatura es escasa en cuanto a las representaciones sociales y construcción de sentido y significados de los adolescentes sobre la maternidad y la paternidad, se encuentran algunos estudios como el de Gómez-Sotelo, Gutiérrez, Izzedin, Sánchez, Herrera y Ballesteros (2012), quienes estudiaron las representaciones sociales de la maternidad en adolescentes bogotanas y concluyeron que en algunos contextos de vulnerabilidad social para las adolescentes y sus familias, el embarazo es considerado como normal porque les permite ganar en estatus social al reconocerse como madres y esposas.

En este contexto surge la pregunta de investigación: ¿Cuáles son las representaciones sociales respecto de la familia, la maternidad, la paternidad y el matrimonio, construidas por un grupo de adolescentes escolarizados en colegios oficiales de Bogotá?, ¿Existen diferencias según edad y sexo en las representaciones sociales que construyen los adolescentes sobre familia, maternidad, paternidad y matrimonio?

Teniendo en cuenta que entre las funciones de la familia, según Pérez-Adán (2005), está la formación de los miembros más jóvenes, la transmisión de la cultura y la procreación, conocer sobre las representaciones sociales que en esta materia elaboran los adolescentes escolarizados en colegios, permite avanzar en la comprensión de la familia desde la vida cotidiana de este grupo poblacional, lo cual se constituye en un aporte a las ciencias sociales.

\section{Método}

En respuesta a las preguntas de investigación, se desarrolló un estudio de tipo descriptivo mixto mediante la aplicación de cuestionario y entrevista.

Para la investigación el grupo de trabajo diseñó dos instrumentos: El primero, un cuestionario compuesto por 18 preguntas teniendo en cuenta variables sociodemográficas, la noción que sobre familia han construido los participantes, el grupo social en el que se sienten más cómodos y seguros, condiciones necesarias para conformar una familia, condiciones necesarias para tener hijos, importancia de la mater- 
nidad/paternidad en el desarrollo de la persona, interés a futuro sobre conformar una familia, interés por ser padre o madre a futuro, edad en la que espera ser padre o madre, número de hijos esperados y creencias sobre el matrimonio como base de la familia.

La variable noción de familia, fue evaluada escogiendo una entre las siguientes opciones: unidad de amor, escuela de aprendizaje para la vida, lugar de la ayuda mutua e incondicional, lugar de refugio, comunidad de personas y lugar de la provisión de recursos.

En cuanto al grupo social en el que se sienten más cómodos y seguros se solicitó escoger una entre las siguientes opciones de respuesta: familia, amigos, novio (a), colegio (maestros y pares).

Respecto de las condiciones previas a la formación de una familia, los participantes podían escoger hasta 3 dentro de las siguientes opciones: terminar los estudios de bachillerato, terminar los estudios universitarios, haber tenido varias experiencias amorosas, haber tenido convivencia en pareja, ser económicamente independiente y tener ingresos suficientes, haber encontrado la persona adecuada, haber logrado madurez, desear tener hijos, amar y comprometerse con la pareja, haber disfrutado la juventud y aún no lo he pensado.

Sobre condiciones necesarias para tener hijos, escogieron hasta 3 entre las siguientes 8 opciones: terminar estudios de bachillerato, terminar estudios universitarios, tener un trabajo estable, ser económicamente independiente/ tener recursos suficientes, poder contar con el apoyo de la familia y los amigos, conocer bien a la pareja, desear tener hijos, que los dos padres así lo quieran y lo decidan, aún no lo he pensado. Esta última opción se presenta entendiendo que el tema aún no hace parte de sus reflexiones personales en marco de su proyecto de vida.

Con relación a la importancia de la maternidad y la paternidad en el desarrollo de las personas, los participantes escogieron 1 entre 3 opciones: sí, no, aún no lo he pensado.

Sobre los planes a futuro de ser padre o madre escogieron 1 entre las opciones: sí, no, aún no lo he pensado. 
Con relación al interés por conformar a futuro una familia, los participantes escogieron 1 entre 3 opciones así: sí, no, aún no lo he pensado.

Respecto de las creencias sobre el matrimonio como base de la familia, los participantes seleccionaron 1 entre las opciones: sí, no, aún no lo he pensado.

La maternidad y la paternidad en el proyecto de vida, fue evaluada sobre las opciones: sí, no, aún no lo he pensado.

Para evaluar la edad óptima para la maternidad y la paternidad, los participantes escogieron en las siguientes alternativas: antes de los 20, después de los 20 años, ya soy padre/madre y aún no lo he pensado.

Sobre el número de hijos esperados, escogieron entre: Uno, entre 2 y 4, más de 4, no tengo planes de tener hijos, aún no lo he pensado.

Ahora bien, el segundo instrumento sirve comoguía de entrevista semiestructurada, con la que se buscó profundizar en vivencias que permite profundizar en la representación social de la maternidad y la paternidad que construye el adolescente. El instrumento se compuso de 5 preguntas teniendo en cuenta variables relativas a la conceptualización, el significado y la vivencia de la maternidad/paternidad adolescente.

Ahora bien, tanto el cuestionario como la guía de entrevista fueron construidos por el grupo de investigadores y para asegurar la validez y confiabilidad de los resultados, siguiendo a Patton (1990), ejecutaron dos tareas: una, centrada en la consulta a expertos, para lo cual fueron sometidos a evaluación de 13 expertos en familia, validez de constructo, contenido y en semántica. Los expertos examinaron la pertinencia de cada ítem y sus correspondientes preguntas, de tal manera que el grupo de preguntas midiera las variables determinadas siguiendo los postulados teóricos de la literatura revisada, buscando asegurar las distintas dimensiones y componentes a evaluar de manera que todos aporten a la comprensión total del tema estudiado y no a otras situaciones asociadas a los adolescentes participantes. El proceso combinó los métodos de Grupo Nominal y de Consenso Grupal (Corral, 2009; Martín 2004). 
La segunda tarea consistió en la prueba piloto (Corral, 2009; Martín, 2004) realizada, en el caso del cuestionario, con un total de 60 participantes y en el caso de la entrevista con 3 adolescentes que pertenecían a la misma población, tarea que determinó el ajuste en la formulación de dos preguntas del cuestionario.

\section{Participantes}

Para la inclusión en la muestra, el diseño determinó como criterios que fueran hombres y mujeres adolescentes, en edades entre los 12 y 18 años matriculados en los grados de sexto a once de 18 colegios de la ciudad de Bogotá, y que previamente fueron determinados.

Una vez revisados uno a uno todos los cuestionarios, la muestra final fue de 2.535 jóvenes participantes con edades entre 12 y 18 años con una $\mathrm{M}=14.49, \mathrm{DE}=1.73$. De ellos el 49.1\%(1.245) son hombres y el 50.9\%(1.290) mujeres. Según el estrato social, el $24.9 \%(632)$ son de estrato bajo, de estrato medio el $24.9 \%(632)$ y el 74.4\%(17) de estrato alto.

En cuanto a la composición familiar, el 56.7\%(1437) de los participantes pertenecen a familias nucleares completas, lo que significa que viven con los dos padres, el 6.1\%(154) con uno de ellos, el 7\%(178) de los participantes hacen parte de una familia reconstituida, es decir que viven con uno de los padres y su nueva pareja. En familias extensa vive el $29.7 \%\left(75^{2}\right)$, es decir, que de su familia hace parte otro familiar como abuelos, tíos, primos. El o.6\%(14) de los adolescentes viven en hogares sustitutos que, para efectos de este estudio, corresponde a los que viven con familias no parientes porque fueron entregados por sus padres a otras familias para ser cuidados o por determinación del Instituto Colombiano de Bienestar Familiar (ICBF), por estar en programas de protección.

Respecto a la edad, el 51.1\%(1295) reportaron edades correspondientes a adolescencia temprana (12,13 y 14), el 35\%(886) a la adolescencia intermedia (15 y 16 años) y el 14\%(354) a la adolescencia avanzada (17 y 18 años). 


\section{Procedimiento}

Durante el primer semestre del año 2015, se gestionó en los colegios el consentimiento informado para la participación de los adolescentes. Seguidamente, se diligenció el cuestionario de manera anónima e individual en línea, por grupos según los grados en cada colegio, y con el acompañamiento del equipo de investigación. Los análisis fueron realizados en el programa SPSS Statistics versión 23. Una vez analizada la información cuantitativa y para profundizar en la comprensión del tema, se desarrollaron 24 entrevistas a adolescentes. La transcripción se realizó en las siguientes 24 horas y la información se organizó en matrices de análisis construidas por el equipo de investigación. Con los análisis de la información recogida mediante los dos instrumentos se realizó un ejercicio de triangulación que permitió la comprensión total del tema.

\section{Resultados}

El proceso de socialización genera de manera continua el legado cultural que, en el caso de la familia, vincula la construcción de las representaciones sociales sobre ser hombre, mujer, madre, padre, entre otras. A continuación se presenta la tabla de convenciones empleada para el análisis de las entrevistasy los resultados que dan respuesta a las preguntas de investigación.

Tabla 1. Convenciones entrevista

\begin{tabular}{lc}
\hline \multicolumn{1}{c}{ CONVENCIONES ENTREVISTA } & SIGLA \\
\hline Adolescente Avanzado Mujer & AAM \\
Adolescente Avanzado Hombre & AAH \\
Adolescente Intermedio Madre & AIM \\
Adolescente Intermedio Padre & AIP \\
Adolescente Avanzado Padre & AAP \\
Adolescente Avanzado Mujer Madre & AAMM \\
\hline
\end{tabular}

Fuente: Autores 


\section{Noción de Familia}

Respecto a la noción de familia, la tabla 2 muestra que la noción de familia de mayor elección es la unidad de amor en los adolescentes en edad temprana, en contraste con los adolescentes en edad avanzada, seguida por lugar de refugio en los participantes en adolescencia intermedia frente a los de adolescencia avanzada. Las opciones con menor elección para los tres grupos son comunidad de personas, seguido por lugar de provisión de recursos.

Tabla 2. Noción de Familia

\section{Participantes}

Noción de Familia

Porcentaje (frecuencia)

\begin{tabular}{lccc}
\cline { 2 - 4 } & Temprana & Intermedia & Avanzada \\
\hline Unidad de amor & $75,1 \%(972)$ & $59,7 \%(529)$ & $54,5 \%(193)$ \\
$\begin{array}{l}\text { Escuela de } \\
\text { aprendizaje para la vida }\end{array}$ & $8,8 \%(114)$ & $11,7 \%(104)$ & $12,7 \%(45)$ \\
$\begin{array}{l}\text { Lugar de ayuda mutua } \\
\text { Lugar de refugio }\end{array}$ & $9,4 \%(122)$ & $19,3 \%(171)$ & $21,5 \%(76)$ \\
$\begin{array}{l}\text { Comunidad de } \\
\text { personas }\end{array}$ & $2,8 \%(36)$ & $4,9 \%(43)$ & $3,4 \%(12)$ \\
$\begin{array}{l}\text { Lugar de provisión de } \\
\text { recursos }\end{array}$ & $2,4 \%(31)$ & $2,8 \%(25)$ & $5,4 \%(19)$ \\
\hline
\end{tabular}

Fuente: Autores

\section{Grupo en el que se sienten más cómodos}

Acerca del grupo en el que se sienten más cómodos y seguros, los adolescentes en los tres rangos de edad consideran que es en la familia $(\mathrm{x} 2(8)=41.96 ; p=.00)$, en contraste el grupo novio $(a)$ que presenta diferencias significativas entre las tres edades. 
Por sexo se encuentran diferencias significativas entre el grupo en el que se sienten más cómodos y seguros $(\mathrm{x} 2(4)=11.42 ; p=.02)$. Para los hombres, es la familia, en contraste con las mujeres que eligieron los amigos. En las entrevistas, uno de los participantes expresó "mi familia siempre me apoyó, cuando mi novia quedo embarazada, a mi mamá fue la que le dio más duro pero ella igual me ayuda” (AAH).

Tabla 3. Grupos en los que se siente más cómodo

\begin{tabular}{lccc}
\hline $\begin{array}{c}\text { Grupo en } \\
\text { el que se } \\
\text { siente más } \\
\text { cómodo }\end{array}$ & Temprana & Porcentaje (frecuencia) & \\
\hline Familia & $85,3 \%(1104)$ & $75,5 \%(669)$ & $76,0 \%(269)$ \\
Amigos & $9,0 \%(117)$ & $14,1 \%(125)$ & $13,8 \%(49)$ \\
Novio/Novia & $2,0 \%(26)$ & $5,0 \%(44)$ & $5,4 \%(19)$ \\
Iglesia & $1,2 \%(16)$ & $2,0 \%(18)$ & $1,7 \%(6)$ \\
Colegio & $2,5 \%(32)$ & $3,4 \%(30)$ & $3,1(11)$ \\
\hline
\end{tabular}

Fuente: Autores

\section{Condiciones necesarias para conformar una familia}

De las 11 opciones que se presentaron para evaluar la variable, como lo muestra la tabla 4, la más escogida fue haber terminado los estudios universitarios. La confirma el hallazgo: "para ser mamá y papá es después que uno termine una carrera, estudie. Uno ya tenga todo" (AAM), "Tener claro lo que uno les va a enseñar y un estudio es muy importante...pero teniendo conciencia que uno tiene un estudio, porque si uno no tiene que ofrecerles también va a ser una dificultad" (AAM).

La segunda condición más seleccionada por los participantes fue haber terminado los estudios de bachillerato y la tercera condición 
con mayor elección corresponde a tener independencia económica. En las entrevistas los adolescentes lo manifestaron así: "Uno debe estar situado en un puesto fijo... establecido lo que va a ser en la vida y tras del hecho tener un buen trabajo, tener todo lo que se pueda para mantener una familia..." (AAP). La menos escogida fue desear tener hijos.

Tabla 4. Condiciones necesarias para conformar una familia

\begin{tabular}{lc}
\hline \multicolumn{1}{c}{ Condiciones } & Porcentaje \\
\cline { 2 - 2 } & Frecuencia \\
\hline 1. Terminar los estudios de bachillerato & $62,8 \%(1592)$ \\
2. Terminar los estudios universitarios & $75,0 \%(1901)$ \\
3. Haber tenido varias experiencias amorosas & $6.0 \%(151)$ \\
4. Haber tenido convivencia en pareja & $11,6 \%(293)$ \\
5. Ser económicamente independiente y tener ingresos suficientes & $46,3 \%(1174)$ \\
6. Haber encontrado a la persona adecuada & $17,3 \%(439)$ \\
7. Haber logrado la madurez & $12,9 \%(326)$ \\
8. Desear tener hijos & $2,6 \%(67)$ \\
9. Amar y comprometerse con la pareja & $9,5 \%(241)$ \\
10. Haber disfrutado la juventud & $9,0 \%(227)$ \\
11. No lo he pensado & $3,7 \%(94)$ \\
\hline
\end{tabular}

Fuente: Autores

\section{Condiciones necesarias para tener hijos}

Frente a las condiciones necesarias para tener hijos, los adolescentes eligieron como prioridad haber terminado los estudios universitarios, seguida por tener un trabajo estable. En las entrevistas los participantes expresaron “... desde que nació el niño, tengo que trabajar para tener cómo responder por las cosas que él necesita... trabajo en las tardes hasta la noche y los fines de semana, porque a la mamá le toca cuidarlo cuando no está estudiando" (AAP). 
Las condiciones con menor elección fueron desear tener hijos y no lo ha pensado.Vale la pena aclarar que esta última opción se presentó teniendo en cuenta que por la edad, algunos adolescentes aún no se lo preguntan. Es decir, aún no se encuentra entre sus reflexiones respecto de su proyecto de vida. En las entrevistas quienes más afirmaron que sí es importante desear tener hijos fueron los adolescentes que ya son padres o madres:“... la comunicación de cuando los dos tengan el deseo de formar una familia sino está seguro, es mejor no hacerlo porque para qué traer una criatura a sufrir a este mundo, si él no tiene la culpa para que después lo dejen solo con la mamá o con el papá e incluso huérfano" (AIP).

Tabla 5 • Condiciones necesarias para tener hijos

\begin{tabular}{lc}
\hline \multicolumn{1}{c}{ Condiciones necesarias para tener hijos } & Porcentaje \\
\cline { 2 - 2 } & (Frecuencia) \\
\hline 1. Terminar los estudios de bachillerato & $49,3 \%(1249)$ \\
2. Terminar los estudios universitarios & $62,2 \%(1578)$ \\
3. Tener un trabajo estable & $49,9 \%(1266)$ \\
4. Ser económicamente independiente / tener ingresos suficientes & $36,0 \%(912)$ \\
5. Poder contar con el apoyo de la familia y los amigos & $17.0 \%(430)$ \\
6. Conocer bien a tu pareja / esposo (a) & $17,8 \%(452)$ \\
7. Desear tener hijos & $6,8 \%(173)$ \\
8. El que los dos padres así lo quieran y lo decidan & $9,5 \%(240)$ \\
9. Aún no lo he pensado & $5,5 \%(139)$ \\
\hline
\end{tabular}

Fuente: Autores

\section{La maternidad y la paternidadcomo parte del desarrollo de la persona}

$\mathrm{Al}$ indagar acerca de si la maternidad y la paternidad hacen parte del desarrollo de la persona, se encuentran resultados significativos $(\mathrm{x} 2(4)=48,47 ; \mathrm{p}=.00)$ entre las edades. Para los adolescentes de los 
tres rangos de edad, prevalece el considerar la maternidad y la paternidad como parte del desarrollo de la persona.

Tabla 6. La maternidad y la paternidad son parte del desarrollo de la persona

La maternidad es parte del desarrollo de la persona

\section{Participantes}

\section{Porcentaje (frecuencia)}

\begin{tabular}{cccc} 
& Temprana & Intermedia & Avanzada \\
\hline Si & $60,6 \%(785)$ & $66,5 \%(590)$ & $69,7 \%(247)$ \\
No & $10,5 \%(137)$ & $14,10 \%(125)$ & $15,8 \%(56)$ \\
No lo he pensado & $28,8 \%(373)$ & $19,3 \%(171)$ & $14,4 \%(51)$ \\
\hline
\end{tabular}

\section{Fuente: Autores}

Interés por la conformación de una familia en el proyecto de vida según edad y sexo

$\mathrm{Al}$ preguntar sobre futuros planes para conformar una familia se observa en las tablas $7 \mathrm{y} 8$ que los hombres participantes tienen mayor interés que las mujeres en conformar una familia. De igual manera, existe una relación significativa $(\mathrm{x} 2(6)=131,7 ; p=, 00)$ entre los participantes que afirman tener en sus planes ser padres frente a los que no lo consideran en los tres rangos de edad. Existe un grupo importante de adolescentes que aún no lo ha contemplado, especialmente en la edad temprana e intermedia. 
Tabla 7. Planes de conformar una familia según sexo

\begin{tabular}{|c|c|c|}
\hline \multirow{2}{*}{$\begin{array}{l}\text { Planes de confor- } \\
\text { mar una familia }\end{array}$} & \multicolumn{2}{|c|}{$\begin{array}{c}\text { Participantes } \\
\text { Porcentaje (frecuencia) }\end{array}$} \\
\hline & Mujeres & Hombres \\
\hline $\mathrm{Si}$ & $70,9 \%(915)$ & 75,7 (943) \\
\hline No & $11,9 \%(154)$ & $8,6 \%(107)$ \\
\hline No lo he pensado & $17,1 \%(221)$ & $15,7 \%(195)$ \\
\hline
\end{tabular}

Fuente: Autores

Tabla 8. Planes de conformar una familia según edad

\begin{tabular}{lccc}
\hline $\begin{array}{c}\text { Planes de } \\
\text { conformar una } \\
\text { familia }\end{array}$ & Temprana & $\begin{array}{c}\text { Participantes } \\
\text { Porcentaje (frecuencia) }\end{array}$ & \\
\hline $\mathrm{Si}$ & $69,6 \%(902)$ & $76,8 \%(681)$ & $77,6 \%(275)$ \\
$\mathrm{No}$ & $10,0 \%(130)$ & $10,0 \%(89)$ & $11,8 \%(42)$ \\
No lo he pensado & $20,3 \%(263)$ & $13,0 \%(116)$ & $10,4 \%(37)$ \\
\hline
\end{tabular}

\section{Fuente: Autores}

\section{La maternidad y la paternidad en el proyecto de vida}

Sobre la idea a futuro de ser padre o madre con relación al sexo, los hombres han pensado más en ser padres con relación a las mujeres. Es importante tener en cuenta que prevalece en las mujeres adolescentes la idea de no ser madre en comparación a los hombres. En cuanto a la edad, en los tres rangos predomina la proyección a ser padre y madre; sin embargo, es importante observar los resultados en las respuestas "Aún no lo he pensado", porque sobresale su elección en los participantes. Los adolescentes que son padres en el momento de responder el cuestionario se ubican con mayor número en el rango de 17 a 18 años (edad avanzada). 
Tabla9. Intención de ser padre o madre según sexo

\begin{tabular}{|c|c|c|}
\hline \multirow{2}{*}{$\begin{array}{l}\text { Intención de ser } \\
\text { padre o madre }\end{array}$} & \multicolumn{2}{|c|}{$\begin{array}{c}\text { Participantes } \\
\text { Porcentaje (frecuencia) }\end{array}$} \\
\hline & Mujeres & Hombres \\
\hline $\mathrm{Si}$ & $37,8 \%(487)$ & $44,3 \%(551)$ \\
\hline No & $32,8 \%(423)$ & $29,9(372)$ \\
\hline Ya lo soy & 3,o \%(39) & $1,4 \%(17)$ \\
\hline Aún no lo he pensado & $26,4 \%(341)$ & $24,5 \%(305)$ \\
\hline
\end{tabular}

Fuente: Autores

Tabla 10. Intención de ser padre o madre según edad

\begin{tabular}{lccc}
\hline $\begin{array}{c}\text { Intención de } \\
\text { ser padre o } \\
\text { madre }\end{array}$ & Temprana & $\begin{array}{c}\text { Participantes } \\
\text { Porcentaje (frecuencia) }\end{array}$ \\
\hline $\mathrm{Si}$ & $38,0 \%(492)$ & $44,1 \%(391)$ & $43,8(155)$ \\
$\mathrm{No}$ & $33,0 \%(427)$ & $30,5 \%(270)$ & $27,7 \%(98)$ \\
Ya lo soy & $0,6 \%(8)$ & $1,5 \%(13)$ & $9,9 \%(35)$ \\
Aún no lo he pen- & $28,4 \%(368)$ & $23,9 \%(212)$ & $18,6 \%(66)$ \\
sado
\end{tabular}

Fuente: Autores

\section{Edad en la que espera ser madre o padre}

Para los adolescentes, la edad que consideran óptima para ser padre o madre es después de los 20 años en contraste con los que consideran edades por debajo de esta. Estos porcentajes son más altos en la adolescencia temprana e intermedia. Las entrevistas lo confirman: "Por ahí a los 22 o 25 porque pues... hay tiempo, hay estudios, en esos tiempos uno puede saber lo que siente el hijo, tiene más conocimientos de los bebés" (AIM), "Yo diría que unos 26 o 28 en donde ya se tiene una estabilidad económica” (AAP). 
Como lo muestra la tabla 11, los participantes en adolescencia temprana eligieron la opción después de los 20 años seguido de los que se encuentran en la adolescencia intermedia y avanzada, aunque en la adolescencia avanzada están los adolescentes que ya se encuentran viviendo la maternidad y paternidad.

Tabla 11. A qué edad espera ser madre o padre

\begin{tabular}{llll}
\hline & & \multicolumn{2}{c}{ Participantes } \\
$\begin{array}{l}\text { A qué edad espera } \\
\text { ser madre o padre }\end{array}$ & Porcentaje (frecuencia) \\
& Temprana & Intermedia & Avanzada \\
\hline Antes de los 20 & $5,8 \%(75)$ & $5,3(47)$ & $5,1 \%(18)$ \\
Después de los 20 & $58,7 \%(760)$ & $63,9 \%(566)$ & $54,0 \%(191)$ \\
Ya soy padre o madre & $0,9 \%(12)$ & $1,7 \%(15)$ & $8,8 \%(31)$ \\
No lo he pensado & $34,6 \%(448)$ & $29,1 \%(258)$ & $32,2 \%(114)$ \\
\hline
\end{tabular}

Fuente: Autores

\section{Número de hijos que piensa tener}

$\mathrm{Al}$ discriminar por sexo la relación es significativa (x2(5) $=46,24$; $p=, 00$ ). Las mujeres opinan que quieren un hijo mientras los hombres prefieren entre 2 y 4 hijos. En las entrevistas expresaron: "a mí no me gustaría llenarme de muchos hijos, la plata no alcanzaría y es más difícil hoy en día cuidarlos" (AAM), "pues sí, yo sé que mi hijo es una bendición, pero ahora que lo tengo veo lo difícil que es ser mamá y yo ya no quisiera tener más” (AAMM). 
Tabla 12. Número de hijos esperados

\begin{tabular}{lcc}
\hline \multirow{2}{*}{ Cuántos hijos piensa tener } & \multicolumn{2}{c}{ Participantes (frecuencia) } \\
\cline { 2 - 3 } & Hombres & Mujeres \\
\hline Uno & $25,4 \%(317)$ & $34 \%(439)$ \\
Entre 2 y 4 & $44,1 \%(550)$ & $33,8(437)$ \\
Más de 4 & $1,8 \%(23)$ & $0,6 \%(9)$ \\
No tengo planes de tener hijos & $5,3 \%(67)$ & $7,8 \%(101)$ \\
Aún no lo he pensado & $23,1 \%(288)$ & $23,5 \%(304)$ \\
\hline
\end{tabular}

Fuente: Autores

\section{El matrimonio como base de la familia}

A la pregunta sobre si el matrimonio es la base de la familia, los hombres a diferencia de las mujeres reportan mayoritariamente que sí como se puede observar en la tabla 13 .

Tabla 13. El matrimonio como base de la familia según sexo

\begin{tabular}{llc}
\hline \multicolumn{2}{c}{$\begin{array}{c}\text { Matrimonio base de la } \\
\text { familia. }\end{array}$} & \multicolumn{2}{c}{ Participantes } \\
& Hombres & Mujeres \\
\hline $\mathrm{Si}$ & $52,5 \%(654)$ & $48,6 \%(627)$ \\
$\mathrm{No}$ & $28,9 \%(360)$ & $30,0 \%(388)$ \\
No lo he pensado & $18,5 \%(231)$ & $21,3 \%(275)$ \\
\hline
\end{tabular}

Fuente: Autores

\section{El matrimonio como base de la familia según edad}

Con relación a las edades, en la tabla 14 se observa que para la etapa temprana, intermedia, y la avanzada, el matrimonio se considera parte 
importante para la familia. Sin embargo, para algunos adolescentes en edad temprana el no considerarlo y el aún no pensarlo no tienen diferencia significativa.

Tabla 14

El matrimonio como base de la familia según edad

\begin{tabular}{llll}
\hline \multicolumn{1}{c}{$\begin{array}{c}\text { Matrimonio base de la } \\
\text { familia. }\end{array}$} & \multicolumn{3}{c}{ Participantes } \\
& Porcentaje (frecuencia) \\
\hline $\mathrm{Si}$ & $52,2 \%(676)$ & $50,5 \%(448)$ & $\begin{array}{l}44,3 \% \\
(157)\end{array}$ \\
No & $23,32 \%(302)$ & $34,0 \%(302)$ & $\begin{array}{l}40,6 \% \\
(144)\end{array}$ \\
No lo he pensado & $24,4 \%(317)$ & $15,3 \%(136)$ & $5,9 \%(53)$ \\
\hline
\end{tabular}

Fuente: Autores

\section{Discusión}

Conocer sobre las representaciones sociales que en esta materia elaboran los adolescentes escolarizados en colegios, permite avanzar en la comprensión de la familia desde la vida cotidiana de los adolescentes.

Los resultados en cuanto a la noción de familia coinciden con los planteamientos de Papalia (2005) cuando argumenta que la relación con los padres se sustenta en la cercanía emocional alcanzada en la niñez, puesto que los lazos que se construyen en las primeras etapas del ciclo de vida se reafirma durante la adolescencia y los factores que proporcionan seguridad facilitan la autonomía, la toma de decisiones y dan significado a la maternidad y la paternidad. En el estudio se evidenció que para los adolescentes en los tres rangos de edad es la familia el lugar donde más cómodo y seguro se sienten, debido a que "buscan en los padres una base segura a partir de la cual puedan probar sus alas de manera muy parecida a lo que hacen los niños pequeños cuando empiezan a explorar un mundo más ancho" (Papalia, p. 404). 
Para los adolescentes el ser padre o madre implica, además de estas situaciones, un cambio en las dinámicas propias de la edad; lo anterior en concordancia con lo que concluye Gómez-Sotelo et al., (2012) de que los adolescentes deben asumir el nuevo rol de padres en la sociedad, generando así múltiples consecuencias como la deserción escolar, la dependencia económica de la familia, temor hacia el rechazo para aquellas jóvenes que esperan su primer bebé, aunque algunas deben postergar sus estudios de secundaria, piensan en culminarlos y continuar una carrera universitaria.

Respecto de los planes futuros sobre conformar una familia y tener hijos, los resultados de esta investigación no coinciden en el grupo de adolescencia temprana con el planteamiento de Unicef (2014) sobre la idealización la maternidad/paternidad como condiciones necesarias de la existencia humana. Sin embargo, a medida que avanza la adolescencia sí reconocen dicha condición.

Para los adolescentes las condiciones necesarias para la maternidad y paternidad se encuentran en haber terminado estudios, tener un trabajo estable y ser independientes económicamente, lo cual puede estar ligado a las transformaciones en el ideal de las mujeres, surgiendo nuevas prioridades asociadas al éxito en otras áreas como las profesionales, tal como lo afirma Llanio (2009).

Los hombres participantes en el estudio reportaron que la paternidad es deseable, resultado que coincide con el planteamiento de $\mathrm{Pu}-$ yana (2003) de que en los hombres surge un interés protagónico en la crianza de los hijos que supera la idea de proveedor y el simbolismo de poder y autoridad. Igualmente, aporta conocimiento en cuanto consultó nuevas cuestiones relacionadas con el interés de conformar una familia, encontrando que los hombres participantes tienen mayor interés que las mujeres en hacerlo.

Los estudios anteriores, no reportan consultas sobre la importancia que le dan los adolescentes al matrimonio como base de la familia, lo cual se constituye en un avance de los resultados de este estudio. 
Los resultados coinciden con Unicef (2014), en cuanto a que es mayor el número de hombres que quieren tener hijos y al consultar sobre el número, igualmente es mayor el número de hombres que quieren más de dos hijos que las mujeres.

Los resultados se alejan de los hallazgos de Nóblega (2009) en cuanto al planteamiento del autor según el cual la maternidad temprana es un recurso de los adolescentes para alejarse de situaciones familiares adversas. Los participantes consideran necesario, antes de conformar una familia, aspectos tales como haber terminado estudios y ser económicamente independientes. Lo mismo sucede con la investigación de Gómez-Sotelo et al. (2012) sobre las representaciones sociales de la maternidad en mujeres adolescentes de Bogotá del que concluyeron que en algunos contextos de vulnerabilidad social, el embarazo les permite reconocerse como madres y esposas. Las participantes de este estudio reportaron menor interés en ser madres que los hombres y como edad esperada indicaron después de los 20 años.

Para los adolescentes participantes del estudio, la familia es una unidad de amor y de ayuda mutua, seguida del lugar de los aprendizajes para la vida, lo cual coincide con la teoría de Moscovici (1979), la cual plantea que la importancia de las representaciones sociales se ubica en la fuerza de las vivencias individuales; el lugar que ocupa en su medio y las situaciones diarias confluyen en la construcción individual en su forma de percibir y vivir el mundo. El autor identifica tres dimensiones que hacen parte de la cultura, organizada por el conjunto de creencias y prácticas que se relacionan con la disposición de los conocimientos que los grupos sociales construyen respecto a un objeto social determinado. Los hallazgos dan cuenta del valor que los adolescentes le atribuyen a la familia desde su experiencia vivida. Esto puede explicar la idea del matrimonio como base de la familia y de las condiciones previas a la conformación de su propia familia y el número de hijos que desean tener. 


\section{Conclusiones}

Para los adolescentes escolarizados en colegios oficiales, la familia representa un valor en cuanto se reconoce como una unidad de amor, lugar del refugio y ayuda mutua. Las representaciones sociales de maternidad y paternidad se encuentran mediadas por el interés de asegurar la independencia y estabilidad económica a partir del trabajo que, a su vez, se garantiza con alcanzar mayores niveles educativos que los que tienen al momento del estudio. Cuestión interesante, teniendo en cuenta que viven en contextos sociales vulnerables.

Las familias de los participantes en su mayoría corresponden al tipo de familia nuclear completa, en donde a pesar del contexto de vulnerabilidad social, prevalece la idea de lugar de refugio, ayuda mutua y unidad de amor. En este sentido, se considera importante retomar resultados obtenidos por sexo y edad frente a los programas de educación de la sexualidad y la afectividad como base para la conformación de sus futuras familias.

A diferencia de las mujeres, los hombres adolescentes de colegios oficiales reportan mayor interés por el matrimonio como base de la familia y la conformación de una familia propia a futuro con más de un hijo.

Los hallazgos observan la vigencia y pertinencia de la teoría sobre las representaciones sociales, en cuanto a las dimensiones que plantea Moscovici (1979) como parte de la cultura y que conforman el universo de creencias que construyen los adolescentes escolarizados en colegios oficiales de Bogotá Colombia.

Realizar futuros estudios que consulten a padres y docentes, se visualiza como un aporte a las ciencias sociales y a la salud pública en tanto se constituyen en una base sólida para formular acciones de intervención frente a la prevención del embarazo adolescente. Igualmente, investigar sobre las representaciones sociales que construyen los adolescentes sobre familia, maternidad, paternidad y matrimonio en instituciones educativas privadas y otros contextos, permitirá avanzar en el conocimiento. 


\section{Referencias}

Alpízar, L., y Bernal, M. (2003). La construcción social de las juventudes. Última década, 11(19), 105-123. Recuperado de: http://www.scielo.cl/pdf/udecada/ v11n19/arto8.pdf

Duarte, J. B. (2013). La relevancia del conocimiento de las representaciones sociales de los adolescentes varones acerca de la paternidad en la adolescencia para el desarrollo de políticas públicas. Revista Subjetividad y Procesos Cognitivos, 17(2), 17-36. Recuperado de: http://dspace.uces.edu.ar:8180/xmlui/ bitstream/handle/123456789/2398/La\%2orelevancia_Barreto-Duarte. pdf?sequence $=1$

Bonfá, Á., Menandro, S., y Cristina, d. M. (2014). A PaternidadeeaMaternidadesob o Olhar de Jovens de ClasseMédia e Baixa: UmEstudoemRepresentaçõesSociais. Revista Colombiana de Psicología, 23(2), 311-324. Recuperado de: http://www.scielo.org.co/scielo.php?script=sci_arttext\&pi$\mathrm{d}=$ So121-54692014000200006

Bronfembrenner, U.(1987). La ecología del desarrollo humano. Experimentos en entornos naturales y diseñados. Ediciones Paidós Iberica.

Castro. L., Rojas, J.A., Pinilla, C.,Docal, M.C., (2015). Construcción del sentido de familia en adolescentes escolarizados. Revista Hojas y Hablas, (12),162-173, ISSN:1794-7030

Cabrera, V., Salazar, P., Docal, M., Aya, V., Ardila, M., Rivera R. (2014). Estilos de vida de los jóvenes y las necesidades de educación sexual. Bogotá: IEMP.

Corral, Y. (2009). Validez y confiabilidad de los instrumentos de investigación para la recolección de datos. Revista ciencias de la educación, (33), 19, . 1-29. Recuperado de: http://recai.uaemex.mx/index.php/recai/article/view/135/o

Donati, P. (2003).Manual de Sociología de la Familia. Pamplona, España: Ediciones Universidad de Navarra.

Donati, P. (2014).La Familia. El genoma de la sociedad. Madrid: Ediciones RIALP 
Erickson, E. (1968). Identity, youth and crisis. New York: Norton.

Galindo Pardo, C. (2012). Análisis del embarazo y la maternidad durante la adolescencia: diferencias socioeconómicas. Desarrollo y sociedad, (69), 133-185. Recuperado de: http://www.scielo.org.co/pdf/dys/n69/n69ao6.pdf

Gómez-Sotelo, Á., Gutiérrez-Malaver, M. E., Izzedin-Bouquet, R., Sánchez-Martínez, L. M., Herrera-Medina, N. E., y Ballesteros-Cabrera, M. (2012). Representaciones sociales del embarazo y la maternidad en adolescentes primigestantes y multigestantes en Bogotá. Rev Salud Pública, 14(2), 189-199.Recuperado de:http://www.scielosp.org/pdf/rsap/v14n2/v14n2ao1

Gómez, I., Molina, R., Zamberlin, N. (2011). Factores relacionados con el embarazo y la maternidad en menores de 15 años en América Latina y el Caribe. Federación Latinoamericana de sociedades de obstetricia y ginecología FLASOG. Lima, Perú. Recuperado de: https://books.google.es/books?hl=es\&lr=\&i$\mathrm{d}=$ sYkXu2AgoPgC\&oi=fn\&pg=PA8\&dq=factores+sociales+en+la+maternida$\mathrm{d}+\mathrm{y}+$ paternidad + adolescente\&ots=fh7GLwpoYE\&sig=GUJPphP1OtPTIrrOS1bkmQIeop4\#v=onepage\&q\&f=true

Galindo Pardo, C. (2012). Análisis del embarazo y la maternidad durante la adolescencia: diferencias socioeconómicas. Desarrollo y sociedad, (69), 133-185., Recuperado de: http://www.scielo.org.co/pdf/dys/n69/n69ao6.pdf Hadjadj, F. (2015). ¿Qué es una familia?Granada. España: Editorial Nuevo Inicio. Lalueza, J., Crespo, I. (2003). Adolescencia y Relaciones Familiares. En Los adolescentes en el siglo XXI: Enfoque psicosocial ONU. Barcelona: Editorial OUC. Recuperado de: https://books.google.com.co/books?id=H86mKlp442sC\&p$\mathrm{g}=\mathrm{PA} 135 \& \mathrm{dq}=$ ciclo+vital+adolescencia\&hl=es-419\&sa=X\&ei=1yWWVcn-HYigyQTUhp2IDQ\&ved=oCBsQ6AEwAA\#v=onepage \&q=ciclo\%20vital\%20adolescencia\&f=false

Lerner, R., Noh, R. E., \& Wilson, C. (1998). The parenting of adolescents and adolescents as parents: A developmental contextual perspective. In Proceedings of 
the conference held in Madison, Wisconsin.Recuperadohttp://parenthood. library.wisc.edu/Lerner/Lerner.html

Llanio, L. Q. (2014). Miremos el Género a través de la Paternidad y la Maternidad. Revista Sexología y Sociedad, 15(41). Recuperado de: http://revsexologiaysociedad.sld.cu/index.php/sexologiaysociedad/article/view/386/428

Maldonado, M.,Micolta, A. (2003). Los nuevos padres, las nuevas madres. Programa Editorial Universidad del Valle. Cali Colombia. Recuperado de: https:// books.google.com.co/books?id=Jp2XFNvogosC\&printsec $=$ frontcover\#v=onepage\&q\&f=false

Martín, M. (2004). Diseño y validación de cuestionarios. Revista Matronas Profesión, 5,(17), 23-29 Recuperado de: http://www.enferpro.com/documentos/ validacion_cuestionarios.pdf

Mardomingo, J. (1994).Psiquiatría del niño y el adolescente ediciones Díaz de Santos Madrid. España. Recuperado de: https://books.google.com. books?id=l1pr$\mathrm{N} 154 \mathrm{iFgC} \&$ printsec $=$ frontcover\&dq=Psiquiatría $+\mathrm{del}+$ niño $+\mathrm{y}+\mathrm{el}+$ adolescente

Moscovici, S. (1979). El Psicoanálisis, su imagen y su público. Editorial Huemul S. A. Buenos Aires. Recuperadode: https://taniars.files.wordpress.com/2008/02/ moscovici-el-psicoanalisis-su-imagen-y-su-publico.pdf

Moscovici, S. (1984). The phenomenon of social representations. En R. M. Farry\& S. Moscovici (Eds.), Social representations (pp. 3-69). Cambridge, Reino Unido: Cambridge UniversityPress.

Mayorga, M. N. (2009). La maternidad en la vida de las adolescentes: implicancias para la acción. Psicología, 27(1),29-54.Recuperado de: http://revistas.pucp. edu.pe/index.php/psicologia/article/view/242/235

Papalia, D.(2005).Psicología del desarrollo. México: Ed. Mc Graw Hill. Novena edición. Patton, M. (1990). Qualitative evaluation and research methods. London, New Delhi: Sage Publications.

Pérez-Adán, J. (2005). Repensar la familia. Madrid: Ediciones internacionales universitarias. 
Puyana, (2003). Padres y madres en cinco ciudades colombianas. Universidad Nacional: Editores Almudena.

Puyana Villamizar, Y., \& Mosquera Rosero, C. (2005). Traer" hijos o hijas al mundo": significados culturales de la paternidad y la maternidad. Revista Latinoamericana de Ciencias Sociales, Niñez y Juventud, 3(2), 111-140.Recuperado de:http://revistaumanizales.cinde.org.co/index.php/RevistaLatinoamericana/article/view/302

Raneri, G.,Wiemann, M. (2007).Social Ecological Predictors of Repeat Adolescent Pregnancy. Recuperado de: http://www.researchgate.net/publication/6452543_Social_ecological_predictors_of_repeat_adolescent_pregnancy

Rodríguez, O. (2012). Representaciones sociales sobre la familia en jóvenes de grados $11^{\circ}$ de algunos colegios públicos y privados de Santa Marta, Colombia. Pensando Psicología, 8(14), 28-38. Recuperado de: http://revistas.ucc.edu.co/index. $\mathrm{php} / \mathrm{pe} /$ article/vi

UNICEF. (2014).Vivencias y relatos sobre el embarazo en adolescentes. Panamá.

Van Dijk, T. (1999).Context models in discourse processing. En H. van Oostendorp \& S. R. Goldman (Eds.). The construction of mental representations during reading (pp. 123-148). Mahwah, NJ: Lawrence Erlbaum. 